\title{
Comparison of the physical fitness traits of Azerbaijan and Iran senior Greco-Roman national wrestling teams
}

\author{
Rahmani F. ${ }^{\mathrm{ABCDE}}$, Mirzaei B. ${ }^{\mathrm{ABCDE}}$ \\ Department of Exercise Physiology, Faculty of Sport Sciences, University of Guilan, Rasht, Iran
}

Authors' Contribution: A - Study design; B - Data collection; C - Statistical analysis; D - Manuscript Preparation; E - Funds Collection.

\begin{abstract}
Purpose: $\quad$ The aim of the present investigation was to compare of the physical fitness traits of Azerbaijan and Iran senior Greco-Roman national wrestling teams.

Material: $\quad$ In this study, 10 elite wrestlers (age 27.7 \pm 3.5 years and training background $8.5 \pm 2$ years) of the senior national wrestling team of Azerbaijan were measured in body composition attributes, muscular strength, muscular endurance, maximal oxygen consumption, flexibility, agility, speed, anaerobic power and explosive power and the results were compared to the national Greco-Roman wrestling team of Iran.

Results: $\quad$ The results of this study, using t-test, showed that the mean of squats $(P=0.013)$ and 40 -yd sprint $(P=0.004)$ in wrestlers of Azerbaijan's team were significantly better than Iran's average norm. However, the values of situps $(P=0.030)$, pull-ups $(P=0.000)$ and $4 \times 9-m$ shuttle run $(P=0.024)$ the mean were significantly lower in the wrestlers of Azerbaijan compared to the norm of Iranian wrestlers.

Conclusions: The results of the physical fitness tests determine the strengths and weaknesses of the training programs and provide practical guidance to coaches in order to assess the extent of wrestlers likely progress or slump.

Keywords: elite, profile, test, body composition, strength, anaerobic power.
\end{abstract}

\section{Introduction}

As one of the heaviest and highest-pressure combat sports, lots of challenges and complications have accompanied wrestling. The interference of different energy systems involved in conducting a wrestling contest on the one hand, and being among weight sensitive sports on the other hand, has made athletes and coaches pay special attention to fitness programs and their undeniable contribution to sporting situations [1]. One of the constant challenges faced by coaches and wrestlers is the availability of information about the physical factors affecting the success of wrestlers [2]. Using physical fitness tests can provide valuable information about the physical characteristics of wrestlers [2].

The implementation of physical fitness tests is a part of the program for assessing the effectiveness of highlevel wrestlers training at international and universal levels. The results of these tests make it possible to identify the weaknesses and strengths of the wrestlers, to recognize the level of differences between them and to conduct necessary planning to overcome weaknesses and deficiencies [3-5]. If these results are measured by relevant norms, they will provide practical guidance to coaches in order to assess the extent of wrestlers' likely progress or slump. Assessing the relative strength and weakness of wrestlers can be a basis for developing a desirable training program [3]. Considering the importance of the issue, a significant part of the studies on the physical fitness of wrestlers has been done on the physiological [6-8] and anthropometric profiles of a wrestling team [9-11] and in some cases only one wrestler $[12,13]$.

Štajer et al. [9] described the physical fitness profile of the Serbian Greco-Roman wrestlers in a study.

\footnotetext{
(c) Rahmani F., Mirzaei B., 2019

doi:10.15561/20755279.2019.0307
}

They measured four variables of maximum oxygen consumption, oxygen consumption at the anaerobic threshold, maximum heart rate and heart rate at the anaerobic threshold. Chaabene et al. [14], in a review article analysed 71 articles in which aerobic and anaerobic characteristics, isometric strength, strength, strengthendurance, and flexibility were measured.

In a study, Arsalanoglu [15] described the physical profile of the young Turkish wrestlers. He measured rest heart rate, flexibility, vertical jumping, 10-20-30m speed, aerobic power, strength, visual and auditory response and fat percentage. Mirzaei et al. [2] described the physiological profile of young wrestlers of the Iranian elite free style team. They measured flexibility (sit and reach test), maximal oxygen consumption, maximal anaerobic leg power, muscular strength and endurance, speed agility and body composition.

In another study, Mirzaei et al. [6] described the physiological profile of Greco-Roman wrestlers of the Iranian senior national team. They measured body composition, relative muscular strength, local muscular endurance, cardio respiratory endurance, speed, agility, flexibility, reaction time, alactic anaerobic power, lactate anaerobic power, and legs explosive power. Rahmani-Nia et al. [16] described the physiological profile of Iranian young Greco-Roman elite wrestlers. They measured the maximum oxygen consumption, muscular endurance, muscle strength, speed, agility and flexibility. Yoon [10] reported that the maximum oxygen consumption of wrestlers participating in international competitions is about $53-56 \mathrm{ml} \cdot \mathrm{kg}^{-1} \mathrm{~min}^{-1}$. He also pointed out that the flexibility of elite wrestlers is higher than wrestlers of lower level. Callan et al. [18] defined the physiological profile of American wrestlers who participated in the preparation of the World Championships 1997 in 6 
physiological parameters. In this study, upper-body muscular power and endurance, body composition, lowerbody muscular power, upper-body power and anaerobic capacity, peak aerobic power (peak $\mathrm{Vo}_{2}$ ), using treadmill or cycle ergometer; and lower back/hamstring flexibility and blood lactate taken 2 minutes after Wingate test and peak $\mathrm{Vo}_{2}$ were measured. They concluded that the physiological and physical fitness profiling of American wrestlers could describe the physical condition of the American wrestlers to provide training approaches to their coaches.

Therefore, considering the necessity of performing physical fitness tests in determining the strengths and weaknesses of the elite wrestlers, the present study aims to describe the physical fitness profile of the GrecoRoman wrestlers of the senior national team of Azerbaijan and compare them to the norms of Iranian national GrecoRoman wrestling team.

\section{Materials and Methods}

Participants

Ten elite wrestlers (age 27.7 \pm 3.5 years and training background $8.5 \pm 2$ years) of the senior national wrestling team of Azerbaijan participated in the study. The participants were selected with European, world and Olympic titles.

Research Design

A descriptive research study was conducted with top senior Greco-Roman wrestlers. The wrestlers were asked not to participate in a daily training program within 24 hours prior to testing and they informed about the tests and study protocols. Testing was completed for all wrestlers in the same place and time of day. Testing and measurements included body composition attributes (fat percentage, lean body mass, body mass index), relative muscular strength (grip force, bench press and squat), local muscular endurance (sit-ups, push-ups and pullups), maximal oxygen consumption (Bruce protocol), flexibility (sit and reach test), agility $(4 \times 9-m$ shuttle run), speed (40-yd sprint), alactic anaerobic power (leg and arm Wingate tests), and explosive power (long jump). All wrestlers were assessed during the general preparation phase of the season.

Statistical Analysis

Before analysis, Normality of the distribution was analyzed using Shapiro-Wilk test. Descriptive statistics were used to calculate the mean and standard deviations of data and to use inferential statistics of single-sample t- test to compare the mean of physical fitness characteristics of two teams. The level of significance for all statistics was set at $p<0.05$. SPSS 25 , software was used to calculate the data.

\section{Results}

Table 1 shows the body composition attributes and table 2 shows the results of the fitness tests of the wrestlers

Table 1. Single-sample t-test results for comparing the body composition attributes of Azerbaijan with Iran

\begin{tabular}{llll}
\hline Body composition attributes & $\begin{array}{l}\text { Mean } \pm \text { Std. Deviation } \\
\text { Azerbaijan }\end{array}$ & Iran & Sig. (2-tailed) \\
\hline Body fat $(\%)$ & $9.01 \pm 4.63$ & $11.3 \pm 3.8$ & -.125 \\
BMI $\left(\mathrm{kg} / \mathrm{m}^{2}\right)$ & $26.5 \pm 4.28$ & $26.9 \pm 4$ & 0.775 \\
LBM $(\mathrm{kg})$ & $73.05 \pm 14.72$ & $71.8 \pm 15.5$ & 0.794 \\
\hline
\end{tabular}

Note: $\mathrm{BMI}=$ Body Mass Index, LBM = Lean Body Mass

Table 2. Single-sample t-test results for comparing the fitness tests of Azerbaijan with Iran

\begin{tabular}{llll}
\hline Fitness tests & $\begin{array}{l}\text { Mean } \pm \text { Std. Deviation } \\
\text { Azerbaijan }\end{array}$ & Iran & Sig. (2-tailed) \\
\hline Grip force $\left(\mathrm{kg}^{-1}\right)$ & $0.91 \pm 0.03$ & $0.91 \pm 0.13$ & 0.761 \\
Bench press $\left({\left.\mathrm{W} . \mathrm{kg}^{-1}\right)}^{1.49 \pm 0.13}\right.$ & $1.42 \pm 0.18$ & 0.119 \\
Squat $(\mathrm{W} . \mathrm{kg}-1)$ & $2.06 \pm 0.31$ & $1.76 \pm 0.26$ & $0.013^{*}$ \\
Sit-ups (n/min) & $67.5 \pm 4.67$ & $71.3 \pm 7.1$ & $0.030^{*}$ \\
Push-ups (n/min) & $66 \pm 4.78$ & $68 \pm 8.7$ & 0.219 \\
Pull-ups (n) & $19.6 \pm 8.34$ & $33.6 \pm 11$ & $0.000^{*}$ \\
Vo max (ml.kg-1 $\left.\mathrm{min}^{-1}\right)$ & $50.24 \pm 3.07$ & $50.4 \pm 5.4$ & 0.878 \\
Sit and reach (cm) & $39 \pm 1.63$ & $39.3 \pm 5.9$ & 0.576 \\
$4 \times 9-m$ shuttle run (s) & $8.81 \pm 0.21$ & $9 \pm 0.42$ & $0.024^{*}$ \\
40-yd sprint (s) & $5.40 \pm 0.23$ & $5.13 \pm 0.21$ & $0.004^{*}$ \\
Arm Wingate (W) & $407.8 \pm 78.85$ & $414 \pm 109.2$ & 0.809 \\
Leg Wingate (W) & $481.9 \pm 70.15$ & $475 \pm 89.4$ & 0.763 \\
Long jump (cm) & $242.7 \pm 6.65$ & $240.2 \pm 16.9$ & 0.265 \\
\hline
\end{tabular}

Note. ${ }^{*} \mathrm{P}<0.05$ 
of Azerbaijan wrestling team and compares it with the average norms of Iranian team.

The results of tables 1 and 2 showed that the mean of squats $(\mathrm{P}=0.013)$ and 40 -yd sprint $(\mathrm{P}=0.004)$ in wrestlers of Azerbaijan's team were significantly better than Iran's average norm. However, the values of sit-ups $(\mathrm{P}=0.030)$, pull-ups $(\mathrm{P}=0.000)$ and $4 \times 9$ meters shuttle run $(\mathrm{P}=0.024)$ the mean were significantly lower in the wrestlers of Azerbaijan compared to the norm of Iranian wrestlers. In other values, there were no significant difference between the wrestlers of the national wrestling team of Azerbaijan and the average norm of Iran's $(\mathrm{P}>0.05)$.

\section{Discussion}

One of the challenges that attracts the attention of coaches and sports experts is identifying the physical factors affecting performance [19]. Access to the physical fitness profile of wrestlers, while describing the existing conditions, will be a criterion for other wrestlers to be evaluated. The results of physical fitness tests, identifies the strengths and weaknesses of the training programs, and provide practical guidance to the coaches in order to assess the extent to which the wrestlers may progress or fall [20]. Therefore, Coaches can help athletes to achieve their peak performance and championship through systematic and scientific planning, without wasting time and money [1].

According to the results of this study, there was no significant difference between fat percentage, body mass index and lean body mass of wrestlers in Azerbaijan and Iran, which matched the findings of the Zaccagni study [21] in fat percentage and body mass index, which was reported as 10.1 and 24.5, respectively. In addition, the result of LBM was consistent with the study by Ratamess et al. [22], which reported a rate of 67.1.

The results of the muscular strength tests indicate that the Azerbaijani wrestlers in the Squat test have a higher mean than Iranian wrestlers, which can be helpful in performing lifts in different techniques. In general, successful wrestlers have more dynamic and static strength than the less successful ones [17].

According to the results of muscular endurance tests, the Azerbaijani wrestlers were higher than Iranian norms in two tests (pull-ups and sit-ups). The results of these two tests were consistent with the study by Chaabene et al. [14], in which they reported a total of 52 repetitions per minute for sit-ups, and more consistent with the pull-ups, which reported 15-50 repetitions.

Based on the findings of this study, the values of the Iranian wrestlers were not significantly different from Azerbaijan team, which is consistent with the findings of Stajer et al. [9] who reported the amount of Vo2max as $50.51 \mathrm{ml} . \mathrm{kg}-1 \mathrm{~min}-1$. In another study, Chaabene et al. [14] reported a mean value of Vo2max of $37-67 \mathrm{ml} . \mathrm{kg}$ $1 \mathrm{~min}-1$, which is consistent with the results of the present study.

Although the changes made to wrestling rules have reduced the aerobic system portion compared with the anaerobic system in energy generation during a wrestling match, the availability of the high Vo2max helps faster and more efficient recovery for wrestlers between two heavy training sessions or two consecutive matches. This is true even about short rests during a wrestling match [1].

In the flexibility test, there was no significant difference in the results of sit and reach test between wrestlers of Azerbaijan and Iran. Which is consistent with the study by Chaabene et al. [14], which reported a 18-45 $\mathrm{cm}$ sit and reach flexibility test. In addition, Yoon [17] reported that the flexibility of elite wrestlers is higher than lower-level wrestlers.

The results of the agility test in a $4 \times 9-m$ shuttle run show that the average of the records of the Azerbaijani wrestlers was less than that of Iran. This excellence can contribute to the rapid implementation of wrestling techniques, which is consistent with Mirzaei et al. [2], who reported a $7.8 \mathrm{~s}$ in a study.

There was no significant difference between the wrestlers of Azerbaijan and Iran in the speed test, 40-yd sprint. Which was higher in the study of Mirzaei et al. [2], which recorded a record of $5.7 \mathrm{~s}$.

No significant difference was observed between the wrestlers of Azerbaijan and Iran in the testing of the alactic anaerobic power and legs explosive power. This feature brings many help to wrestlers in the implementation of speedy techniques and quick reactions. High anaerobic power and legs explosive power are essential for the success in performing techniques such as high dive or lifting opponents like Reverse lift [12].

\section{Conclusion}

In general, considering that the analysis of physical fitness tests in both teams of Azerbaijan and Iran shows that they are both efficient, and the results are constant with other studies conducted on elite athletes, but with it cannot be claimed so assertively that the only factor affecting the performance of Azerbaijani and Iranian wrestlers, who have always been among the world's top teams, is physical fitness. Although having a high physical fitness, from a theoretical point of view, can be related to sporting success, but in no way guarantees a definitive victory; but it is just a ring of championship chains. Other rings of this chain also more or less influence the process of the championship. Some of the most important factors affecting this are; the coaches' knowledge of the correct implementation of the principles of training, proper training, proper technique and tactics, correct assessment of the opponent, nutrition and correct weight control, sleep and rest, illnesses and injuries, psychological and stress factors, team management and leadership, the importance of the result of the match, the proper management of participating in preparatory matches, and so on. Although the importance of all of the abovementioned factors is not the same, however, in order to achieve consistent success in the international level, all the above factors should be considered. 


\section{Acknowledgements}

The authors would like to thank the study participant for their dedication and effort throughout the study.

\section{Conflict of interest}

The authors declare that there is no conflict of interest.

\section{References}

1. Mirzaei B, Rahmani F. Principles of planning and designing wrestling. First ed. Tehran: Hatmi; 2019.

2. Mirzaei B, Curby DG, Rahmani-Nia F, Moghadasi M. Physiological profile of elite Iranian junior freestyle wrestlers. The Journal of Strength \& Conditioning Research. 2009;23(8):2339- 44. https://doi.org/10.1519/JSC.0b013e3181bb7350

3. Bayati R, Majelan AS, Mirzaei B, Barbas I. The Effect of 12 Weeks of Wrestling+ Warm-Up Program on Functional Movement Screen Scores in Cadet Wrestlers. Annals of Applied Sport Science. 2019;7(1):39-47. https://doi.org/10.29252/aassjournal.7.1.39

4. Jagiello W. Differentiation of the body composition in taekwondo-ITF competitors of the men's Polish national team and direct based athletes. Archives of Budo. 2015;11:329338.

5. Kalina RM, Jagiello W. Non-apparatus, Quasi-apparatus and Simulations Tests in Diagnosis Positive Health and Survival Abilities. In: Ahram T, editor. Advances in Human Factors in Sports, Injury Prevention and Outdoor Recreation, Ahfe 2017. Advances in Intelligent Systems and Computing. 6032018. p. 121-128. https://doi.org/10.1007/978-3-319-60822-8_12

6. Mirzaei B, Ghafouri A. Physiological profile of the wrestlers of the senior national team. The Journal of Sports Physiology Research, 2007 Jun 22;3(5):57-66

7. Saad AH. Physiological profile of the young Egyptian wrestlers. World Journal of Sport Sciences. 2012;6(1):45-50.

8. Starosta W, Rynkiewicz T. Test battery for the evaluation and assessment of movement abilities in elite polish wrestlers. International Journal of Wrestling Science. 2014;4(1):49-55. https://doi.org/10.1080/21615667.2014.10879000

9. Štajer V, Trivic T, Rocklicer R, Madic D, Ostojic S, Drid P. Physical condition profile of Serbian Greco-Roman style wrestlers. InInternational scientific and professional conference on wrestling: Applicable research in wrestling, 2017. P. 146-152.

10. Yamashita D, Arakawa H, Arimitsu T, Wada T, Yumoto K, Fujiyama K, Nagami T, Shimizu S. Physiological Profiles of International-and Collegiate-Level Japanese Male Freestyle Wrestlers in the Lightweight Classes. International Journal of Wrestling Science. 2017;7(1-2):21--5. https://doi.org/10.1080/21615667.2017.1341572

11.Zi-Hong H, Lian-Shi F, Hao-Jie Z, Kui-Yuan X, Feng-Tang C, Da-Lang T, Ming-Yi L, Lucia A, Fleck SJ. Physiological profile of elite Chinese female wrestlers. The Journal of Strength \& Conditioning Research. 2013;27(9):2374-95. https://doi.org/10.1519/JSC.0b013e31827f543c
12.Mirzaei B, Curby DG, Barbas I, Lotfi N. Anthropometric and physical fitness traits of four-time World Greco- Roman wrestling champion in relation to national norms: A case study. Journal of Human Sport and Exercise. 2011;6(2):406-413. https://doi.org/10.4100/jhse.2011.62.21

13.Utter AC, O’Bryant HS, Haff GG, Trone GA. Physiological Profile of an Elite Free Style Wrestlers Preparing for Competition:ACase Study. JStrength Cond Res. 2002.308-15. https://doi.org/10.1519/00124278-200205000-00022

14.Chaabene H, Negra Y, Bouguezzi R, Mkaouer B, Franchini E, Julio U, Hachana Y. Physical and physiological attributes of wrestlers: an update. The Journal of Strength \& Conditioning Research. 2017;31(5):1411-42. https://doi.org/10.1519/JSC.0000000000001738

15.Arslanoglu E. Physical profiles of Turkish young GrecoRoman wrestlers. Educational Research and Reviews. 2015;10(8):1034-8.

16.Rahmani-Nia F, Mirzaei B, Nuri R. Physiological profile of elite Iranian junior Greco-Roman wrestlers. International Journal of Fitness, 2007;3(2): 2339 - 2344

17.Yoon J. Physiological Profiles of Elite Senior Wrestlers. Sports Medicine, 2002;32:225-33. https://doi. org/10.2165/00007256-200232040-00002

18.Callan SD, Brunner DM, Devolve KL, Mulligan SE, Hesson J, Wilber RL, Kearney JT. Physiological profiles of elite freestyle wrestlers. The Journal of Strength \& Conditioning Research. 2000;14(2):162-9. https://doi.org/10.1519/00124278-200005000-00008

19. Mirzaei B, Rahmini-nia F, Ghahremani Moghaddam M. A comparative study of body composition, aerobic power, anaerobic power and srenght of Iranian freestyle and GrecoRoman wrestlers participating in the Beijing Olympic Games 2008. Journal of sports science, Exercise \& society. 2010; 49(1):192-194.

20.Mirzaei B, Mansour Sadeghi M. The profile of physical fitness of the adult wrestlers taking part in national team of freestyle wrestling preparation expeditions. The Journal of Olympics, 2006;15(38):81-89.

21.Zaccagni L. Anthropometric characteristics and body composition of Italian national wrestlers. European Journal of Sport Science. 2012;12(2):145-51. https://doi.org/10.1080/17461391.2010.545838

22.Ratamess NA, Hoffman JR, Kraemer WJ, Ross RE, Tranchina CP, Rashti SL, Kelly NA, Vingren JL, Kang J, Faigenbaum AD. Effects of a competitive wrestling season on body composition, endocrine markers, and anaerobic exercise performance in NCAA collegiate wrestlers. European Journal of Applied Physiology. 2013;113(5):1157-68. https://doi.org/10.1007/s00421-012-2520-8 
Information about the authors:

Rahmani F.: (Corresponding author); http://orcid.org/0000-0001-7570-0026: m.foadrahmani@yahoo.com: University of Guilan: Rasht, Khalij Fars highway, 5th kilo meter of Ghazvin road, Postal Code 4199613776, Iran.:

Mirzaei B.: http://orcid.org/0000-0003-3723-7434: bmirzaei2000@yahoo.com:University of Guilan: Rasht, Khalij Fars highway, 5th kilo meter of Ghazvin road, Postal Code 4199613776, Iran.

\section{Cite this article as:}

Rahmani F , Mirzaei B. Comparison of the physical fitness traits of Azerbaijan and Iran senior Greco-Roman national wrestling teams. Physical education of students, 2019;23(3):155-159.

https://doi.org/10.15561/20755279.2019.0307

This is an Open Access article distributed under the terms of the Creative Commons Attribution License, which permits unrestricted use, distribution, and reproduction in any medium, provided the original work is properly cited http://creativecommons.org/licenses/by/4.0/deed.en

Received: 15.04.2019

Accepted: 20.05.2019; Published: 28.06.2019 